\title{
Temporary clamping of drain combined with tranexamic acid reduce blood loss after total knee arthroplasty: a prospective randomized controlled trial
}

\author{
Keerati Chareancholvanich", Pichet Siriwattanasakul, Rapeepat Narkbunnam and \\ Chaturong Pornrattanamaneewong
}

\begin{abstract}
Background: Total knee arthroplasty (TKA) is associated with a significant blood loss. Several methods have been reported to reduce postoperative blood loss and avoid homologous blood transfusions. In this study, we investigated the efficacy of temporary clamping of the drain either or not in combination with tranexamic acid administration for controlling blood loss after TKA.

Methods: The prospective, randomized, and double-blinded study was conducted in our institute. Total of 240 patients, who diagnosed primary osteoarthritis and scheduled to undergo a primary TKA, were randomized into one of the four groups: Group A or control group, the drain was not clamped and the patient received a placebo; Group B, the drain was not clamped and the patient received tranexamic acid; Group C, the drain was clamped and the patient received a placebo; and Group D, the drain was clamped and the patient received tranexamic acid. The volume of drained blood at 48 hours postoperatively, the decreasing of hemoglobin ( $\mathrm{Hb}$ ) level at 12 hours postoperatively and the number of patients requiring blood transfusion were recorded and compared.

Results: The mean postoperative volumes of drained blood and the amount of blood transfusion in the three study groups (group B, C and D) were significantly lower than those in the control group $(p<0.05)$, which group $D$ had the lowest values. Furthermore, group B and D could maintain the Hb level better than group A and $C$ $(p<0.001)$. In terms of blood transfusions rate, although the patients in group $D$ required transfusion less than group $A$ and $C(p<0.05)$, there was no significant difference between group $D$ and $B$. The relative risks for transfusion requirement were 4.4 for group A, 1.4 for group B and 3.0 for group C when compared to group D. Conclusions: The clamping of drain combined with tranexamic acid administration could reduce postoperative blood loss and blood transfusion after TKA, significantly greater than using tranexamic acid or drain clamping alone.

Trial registration: ClinicalTrials.gov NCT01449552

Keywords: Total knee arthroplasty, Bleeding, Drain, Clamp, Tranexamic acid
\end{abstract}

\footnotetext{
* Correspondence: keeratic@hotmail.com

Department of Orthopedic Surgery, Faculty of Medicine, Siriraj Hospital,

Mahidol University, Bangkok, Thailand
} 


\section{Background}

Total knee arthroplasty (TKA) is one of the effective treatments for severe osteoarthritic knee. The goals of this procedure include correcting deformity, restoring pain-free motion and maintaining stability of the knee. Currently, TKA is widely acknowledged to be one of the most successful and cost-effective procedures in orthopedic practice. However, considerable blood loss after TKA is still problematic and postoperative blood transfusion carries a substantial risk of both immunologic reaction and transmission of diseases [1]. Blood transfusion also involves an additional cost, so a reduction in its use is very important.

Several methods have been reported to reduce postoperative blood loss and avoid homologous blood transfusion. The methods shown to be effective include autologous blood transfusion [2], postoperative blood salvage [3-5], intramedullary femoral plug [6], hypotensive anesthesia [7], cryotherapy with Robert Jones bandage [8], fibrin tissue adhesive $[9,10]$, drain clamping [11-18], and tranexamic acid administration [19-29].

Although most of the surgeons performed TKAs with the use of tourniquet, postoperative blood loss still occurred. Most of the blood loss in TKA occurs during the first few postoperative hours [30]. The drain clamping is one of the methods proposed to reduce blood loss in early postoperative period of TKA. Various protocols of this method have been reported [11-18]. For examples, Sakihara et al. [11] infused $50 \mathrm{ml}$ of saline containing antibiotic into the knee joint through the drain tube and clamped the drain for 1 hour. Shen et al. [15] clamped the drain for 4 hours. Tsumara et al. [16] clamped the drain for 30 minutes with intra-articular injection of saline with adrenaline. Prasad et al. [17] recommended using 2-hour clamping of the drain and release for 10 minutes. All of these previous studies proved that the uses of their methods could reduce blood loss and transfusion requirement following TKA. Nevertheless, some studies reported that drain clamping had no benefit in routine TKA [31].

Tranexamic acid is a synthetic anti-fibrinolytic drug used to prevent bleeding. Initially, fibrinolysis is stimulated by surgical trauma [32,33] and further augmented by the use of a tourniquet $[34,35]$. This phenomenon may lead to increase blood loss after TKA, especially during the first few postoperative hours. Administration of tranexamic acid can inhibit the activation of plasminogen to plasmin $[36,37]$ by blocking the lysine binding sites of plasminogen to fibrin, which results in inhibition of fibrinolysis [38,39].

Several studies reported that tranexamic acid could reduce either blood loss or blood transfusions [19-23]. While some studies demonstrated its efficacy in reducing blood loss but not reducing blood transfusion [24,25]. Hynes et al. [26] found that tranexamic acid also could reduce the decreasing of hemoglobin $(\mathrm{Hb})$ level following TKA. Nevertheless, Engel et al. [40] revealed that tranexamic acid did not cause a significant modulation of fibrinolysis variables or a significant reduction of postoperative bleeding and transfusion requirements.

Based on the level I evidences, meta-analyses showed that intravenous tranexamic acid appears effective and safe in reducing allogeneic blood transfusion and blood loss in TKA without increasing the risk of thromboembolic complications $[28,29]$. To our knowledge, no study has compared the efficacy of drain clamping combined with tranexamic acid administration in the control of bleeding following TKA. Thus, the purpose of this study was to evaluate the efficacy of drain clamping alone, tranexamic acid alone and the combination of these two modalities in the control of bleeding following TKA.

\section{Methods}

This study was designed as a prospective, randomized, double-blinded, controlled trial and approved by the Ethics committee of Siriraj Hospital. All patients gave their written informed consent for participation in the study. Between June and November 2008, 240 patients who scheduled to undergo a unilateral primary TKA were enrolled in the study. The patients aged less than 85 years and diagnosed primary osteoarthritic knees were included. The exclusion criteria were the patients who had secondary osteoarthritis (such as rheumatoid arthritis, post-traumatic arthritis, gouty arthritis, postseptic arthritis), high risk medical co-morbidity, history of thromboembolic disease, bleeding disorder, known allergy to tranexamic acid, and receiving the anti-coagulant drugs.

A computer-generated list was created by the blocksof-12 randomization method. The patients were randomly assigned to one of the four groups: group A was non-clamping of drain and placebo administration, group $\mathrm{B}$ was non-clamping of drain and tranexamic acid administration, group $\mathrm{C}$ was clamping of drain and placebo administration and group D was clamping of drain and tranexamic acid administration. The concealed envelopes were used to blind the surgeon and participants for the randomization sequences and opened by a second year resident, who was not involved in the study.

Our senior author $(\mathrm{KC})$, who is experienced in TKA, performed all of the operations on patients who were under spinal anesthesia. A pneumatic tourniquet with a pressure of $350 \mathrm{mmHg}$ was inflated after limb exsanguinations. A straight skin incision and a mini-medial parapatellar capsular incision were used in all knees. The 
patellas were not resurfaced. A Nexgen LPS-Flex fixedbearing design (Zimmer, Warsaw, Indiana), inserted with cement, was used. An intramedullary femoral alignment rod was used in all cases, and the femoral canal was filled with a bone plug before the prosthetic implantation.

In group $\mathrm{B}$ and $\mathrm{D}$ that received tranexamic acid protocol, $10 \mathrm{mg} / \mathrm{kg}$ of intravenous tranexamic acid (Transamin; $250 \mathrm{mg} / 5 \mathrm{ml}$, OLIC, Thailand) was administrated at 10 minutes before inflating the tourniquet and $10 \mathrm{mg} / \mathrm{kg}$ at 3 hours postoperatively. Then, $1500 \mathrm{mg}$ per day of oral-formed tranexamic acid (Transamin; $250 \mathrm{mg} / \mathrm{capsule}$, OLIC, Thailand) was given for 5 days after operation. While in group $\mathrm{A}$ and $\mathrm{C}$, the placebo (equivalent volume of physiologic saline combined with a starch capsule) was administrated instead.

At the end of the operation, a number-10-gauge drain was placed intra-articularly and was connected to the Ultravak pressure drainage bottle (Poly Medicure Limited, India). After wound closure, a compressive Robert Jones bandage and a posterior splint were applied. Following the release of tourniquet pressure, the drain in group A and B was immediately released and the drain in group $C$ and $D$ was clamped following our protocol, so called 3-hourinterval clamping technique (clamped for 3 hours, released for 3 hours, reclamped for 3 hours and then the clamp was run continuously).

The patient was encouraged to perform a mechanical ankle pumping exercise to prevent deep vein thrombosis as soon as possible after surgery. The bandage, splint and Foley catheter were removed on the first post- operative day. On the same day, the range-of-motion exercise, an isometric/isotonic quadriceps exercise, a straight leg rising exercise and a walking exercise were initiated under the control of a physiotherapist.

The amount of drained blood was recorded at $48 \mathrm{hrs}$. All suction drains were removed 48 hours postoperatively. The Hb levels were determined preoperatively and 12 hours postoperatively. The patients received a transfusion of one unit of packed red cells (PRC), if their $\mathrm{Hb}$ levels decreased to $<10 \mathrm{~g} / \mathrm{dL}$ or if the compromised clinical criteria (e.g., tachycardia, hypotension, or symptoms of anemia that were relative to the preoperative medical condition of the patient) necessitated transfusion. If $\mathrm{Hb}$ levels decreased to $<8 \mathrm{~g} / \mathrm{dL}$, the patient received two units of PRC. Following the blood transfusions, we reevaluated $\mathrm{Hb}$ levels at 6 hours after the end of the transfusion period, and blood replacement was considered again using the same criteria as outlined above. At 48 hours after the operation, the $\mathrm{Hb}$ levels of all patients were recorded. Clinical thromboembolic events and wound complications were also examined. All of the patients were discharged from the hospital on the fifth day after the operation.

\section{Statistical analysis}

The data were analyzed using the commercially available SPSS statistics software, version 13.0. Quantitative data were presented as the mean \pm standard deviation and differences in the means among the four groups were analyzed using an analysis of variance (ANOVA). We determined differences in sex and the number of patients requiring blood transfusions using the Chisquare test. The risks of blood transfusion compared between groups were analyzed using logistic regression. $\mathrm{P}$ values $<0.05$ were regarded as statistically significant.

\section{Results}

Over a six-month period, 240 patients (60 patients per group) were available for analysis. The mean age of the patients was $69.8 \pm 6.8$ (range from 53-84) years. The preoperative data included age, gender and preoperative $\mathrm{Hb}$ were comparable among the four groups (Table 1). The mean postoperative volumes of drained blood were $1182 \pm 411 \mathrm{ml}$ in group A, $724 \pm 246 \mathrm{ml}$ in group B, $821 \pm 337 \mathrm{ml}$ in group C, and $526 \pm 222 \mathrm{ml}$ in group D. Group A had significantly higher volume of drained blood than the others $(\mathrm{p}<0.001)$ but there were no significant differences in those between group $B$ and $C$ $(p=0.37)$. The lowest drained-blood volume was found in group $\mathrm{D}(\mathrm{p}<0.001)$ (Table 2$)$.

The mean levels of decreasing $\mathrm{Hb}$ at postoperative 12 hours were $3.3 \pm 0.9,2.1 \pm 0.6,2.8 \pm 0.8$ and $1.8 \pm 0.7 \mathrm{~g} / \mathrm{dl}$ in group A, B, C and D, respectively. Group B, C and D had significantly lower levels of decreasing $\mathrm{Hb}$ than those in group A $(p<0.05)$. Group B and D could maintain the $\mathrm{Hb}$ level better than group $\mathrm{C}(\mathrm{p}<0.001)$, however; there was no significant difference between group $\mathrm{D}$ and $\mathrm{B}(\mathrm{p}=0.07)$ (Table 2).

The amount of PRC transfusion units required in group $\mathrm{B}$ and $\mathrm{D}$ were significantly lower than group $\mathrm{A}$ and $C(p<0.001)$. There was no significant difference between group $A$ and $C(p=0.05)$. The patients who received the least blood transfusion $(0.4 \pm 0.5$ unit) were in group D (significantly lower than group $\mathrm{B}, \mathrm{p}<0.04$ ) (Table 2). In the number of patients requiring blood transfusion, group $\mathrm{D}$ had significantly lower number than group $A$ and $C(p<0.05)$. However there were no significant difference between group D and B (Table 2).

\section{Table 1 Pre-operative data}

\begin{tabular}{llllll}
\hline Characteristics & Group A & Group B & Group C & Group D & p-value \\
\hline & $(n=60)$ & $(n=60)$ & $(n=60)$ & $(n=60)$ & \\
Male : Female & $8: 52$ & $9: 51$ & $10: 50$ & $8: 52$ & 0.946 \\
Age $(y r)^{*}$ & $69.8 \pm 6.3$ & $69.4 \pm 6.3$ & $68.9 \pm 7.5$ & $70.1 \pm 7.2$ & 0.418 \\
Pre-op Hb $(\mathrm{g} / \mathrm{dl})^{*}$ & $12.5 \pm 1.1$ & $12.4 \pm 1.1$ & $12.4 \pm 1.1$ & $12.6 \pm 1.0$ & 0.515 \\
\hline
\end{tabular}

* Data was presented as mean \pm standard deviation. Pre-op $\mathrm{Hb}=$ Preoperative hemoglobin. 
Table 2 Blood loss and transfusion requirement

\begin{tabular}{|c|c|c|c|c|c|}
\hline Values & Group A & Group B & Group C & Group D & p-value \\
\hline & $(n=60)$ & $(n=60)$ & $(n=60)$ & $(n=60)$ & \\
\hline Volume of drained blood & $1182 \pm 411$ & $724 \pm 246$ & $821 \pm 337$ & $526 \pm 222$ & $<0.001^{a}$ \\
\hline$(m l)^{*}$ & $(420-2260)$ & $(230-1330)$ & $(230-2020)$ & $(180-960)$ & \\
\hline Decreasing $\mathrm{Hb}$ at 12 hours & $3.3 \pm 0.9$ & $2.1 \pm 0.6$ & $2.8 \pm 0.8$ & $1.8 \pm 0.7$ & $<0.05^{\mathrm{b}}$ \\
\hline$(g / d l)^{*}$ & $(1.3-5.3)$ & $(0.9-3.8)$ & $(0.9-4.4)$ & $(0.4-3.6)$ & \\
\hline Number of patients requiring blood transfusion & $53(88.3 \%)$ & $34(56.7 \%)$ & $49(81.7 \%)$ & $23(38.3 \%)$ & $<0.05^{c}$ \\
\hline \multirow[t]{2}{*}{ PRC transfusion (unit) ${ }^{*}$} & $1.8 \pm 1.0$ & $0.7 \pm 0.7$ & $1.3 \pm 0.9$ & $0.4 \pm 0.5$ & $<0.05^{\mathrm{d}}$ \\
\hline & $(0-4)$ & $(0-2)$ & $(0-3)$ & $(0-2)$ & \\
\hline
\end{tabular}

* Data was presented as mean \pm standard deviation and range.

a Statistical difference among all groups (except between group $B$ and $C, p=0.37$ ).

${ }^{b}$ Statistical difference among all groups (except between group $B$ and $D, p=0.07$ ).

c Statistical difference among all groups (except between group $A$ and $C, B$ and D).

${ }^{d}$ Statistical difference among all groups (except between group $A$ and $C, p=0.05$ ).

$\mathrm{Hb}=$ hemoglobin, $\mathrm{PRC}=$ packed red cell.

The relative risks for transfusion requirement were 4.4 for group A, 1.4 for group B and 3.0 for group C when compared to group D.

In terms of complications, no wound infections or clinical venous thromboembolisms were detected in all groups. Although three patients (each one from group A, B and D) developed postoperative ecchymosis around the knees, this resolved spontaneously.

\section{Discussion}

The significant blood loss and risk for blood transfusion are important features that must be considered in TKA. Complications after allogeneic blood transfusions have been well reported in the previous literature [1]. Several methods have been reported to reduce blood loss and blood transfusion after TKA [2-29]. However, the best method remains unknown. Either drain clamping [11-18] or tranexamic acid administration [19-29] is the simple method that we interested. The combined effect of these two methods was still unknown. We therefore aimed to study the efficacy of drain clamping alone, tranexamic acid alone and the combination of these two modalities in the control of bleeding following TKA.

Because most of the blood loss in TKA occurs during the first postoperative day ( 71.1 and $84 \%$ in the first 6 and 12 hour after operation, respectively) [41,42], it seems reasonable to clamp the drain in the early postoperative period to create a tamponade effect and to control blood loss. Although various protocols for drain clamping have been reported in the literature [11-18], we have established a new interval clamping protocol for reducing blood loss in TKA. Kiely et al. [31] concluded that 2 hours of drain clamping has no benefit in routine TKA. Thus, a longer period of drain clamping may be required. However, hematoma and wound complications must be taken into consideration for long-period clamping protocols. To balance between creating a tamponade effect and reducing wound complications, an interval clamping protocol using a 3-hour interval pattern that routinely used in our institute was selected for this study. From the results of this study, our drain clamping technique alone could reduce more blood loss and keep higher 12-hour $\mathrm{Hb}$ level than the non-clamping group. Nevertheless, the use of this protocol did not affect the reduction of transfusion requirement, either amount or rate.

Fron non-pharmacological to pharmacological method, there are four routes for administrating tranexamic acid in order to reduce blood loss in TKA: oral, intramuscular, intravenous, and intra-articular [27]. The time taken for maximum plasma levels of tranexamic acid to be reached has been reported to be 2 hours for oral, $30 \mathrm{~min}$ utes for intramuscular and 5-15 minutes for intravenous administration [43,44]. Many clinical studies reported tranexamic acid reduced blood loss or transfusion requirements when given on deflation of the tourniquet with a repeated dose postoperatively [19,20,22-29,45]. Tanaka et al. [27] concluded that the hemostatic effect was best when tranexamic acid was given once $10 \mathrm{~min}$ utes before surgery and once upon deflation of the tourniquet. The administration before the operation gave more hemostatic effect than administration upon deflation of the tourniquet. Pharmacokinetic studies $[43,44,46,47]$ indicated that a dose of $20 \mathrm{mg} / \mathrm{kg}$ of tranexamic acid is suitable for TKA since therapeutic levels could be maintained for approximately 8 hours after the operation, which covered the period of hyperfibrinolysis in cases of increased blood loss. Thus, we also used a dose of $20 \mathrm{mg} / \mathrm{kg}$ of intravenous tranexamic acid that divided into two parts: $10 \mathrm{mg} / \mathrm{kg}$ at 10 minutes before tourniquet inflation and another $10 \mathrm{mg} / \mathrm{kg}$ at 3 hours postoperatively. Then, an oral form of tranexamic acid was given for 5 days in order to control bleeding during rehabilitative training. 
With using our tranexamic acid regimen, we found that it significantly reduced about $40 \%$ of blood loss, compared to those in control group. This result was in the same way of previous studies that reported on 30-50\% of blood loss reduction [19-24,27]. Furthermore, our protocol also showed the superior efficacy in reducing amount and rate of blood transfusion, and maintaining the $\mathrm{Hb}$ level, over the control group.

The hemostatic effects of the tranexamic acid alone were significantly better than the drain clamping alone with regards to the level of decreasing $\mathrm{Hb}$, amount of blood transfusion and number of patients requiring blood transfusion, despite the volume of drained blood between these two methods was not significantly different. These findings confirmed that some blood might remain around the knee joint, leak through the wound, or diffuse into the soft tissue, especially when the drain was clamped $[13,48]$.

Importantly, this study also verified the efficacy of the administration of tranexamic acid combined with the drain clamping, which had never been studied before. Compared to the control group, the use of this combination could reduce the volume of drained blood up to $55 \%$ that significantly more than using the tranexamic acid alone (40\% of reducing blood loss) or the drain clamping alone (30\% of reducing blood loss). Furthermore, this combined method provided the lowest amount of transfusion unit. For the efficacy in maintaining the $\mathrm{Hb}$ level and reducing the rate of transfusion requirement, the use of our tranxenamic acid protocol combined with the 3-hour interval drain clamping was proved to give more benefit than using drain clamping alone. Nevertheless, it was not significantly different from using tranexamic acid alone. The superior effect of tranexamic acid over clamping of the drain might explain this phenomenon. After calculating the relative risks, the number of patients required blood transfusion in control group was 4.4-fold, in drain clamping alone was 3.0-fold and in tranexamic acid alone was 1.4-fold when compared to the combined method.

There are some limitations in this study. First, the female to male ratio was high because most patients undergoing TKA in our country are females. Female patients may have less preoperative hemoglobin than male, which may affect the rate of blood transfusion. Nevertheless, after randomization, the female-to-male ratios and preoperative hemoglobin levels were not different among the four groups. Second, we used only clinical evaluations to evaluate the thromboembolic complications that could not detect asymptomatic deep vein thrombosis and pulmonary embolism. The correlation of using tranexamic acid and venous thromboembolism remains unknown. Third, although we proposed a 5-day regimen of oral formed tranexamic acid might assist to control blood loss during rehabilitation, there were no measurement tools to assess this hypothesis. Finally, we focused only on the efficacy in controlling blood loss. This report did not include functional scoring systems or patient satisfaction.

\section{Conclusion}

This study proved that 3-hour-interval drain clamping technique significantly reduced postoperative blood loss and our tranexamic acid administration protocol significantly reduced blood loss and blood transfusion after TKA. The use of these two combined methods demonstrated the better hemostatic effect than using tranexamic acid alone or drain clamping alone.

\section{Competing interests}

The authors declare that they have no competing interests.

\section{Authors' contributions}

KC made substantial contributions to conception and design, data acquisition, data analysis, and data interpretation. PS made substantial contributions to conception and design, data acquisition, data analysis, data interpretation and drafting the manuscript. RN and CP made data analysis, data interpretation, drafting the manuscript and revising it critically for important intellectual content. All authors read and approved the final manuscript.

\section{Authors'information}

Associate Prof. Keerati Chareancholvanich, MD.; Pichet Siriwattanasakul, MD.; Rapeepat Narkbunnam, MD. and Chaturong Pornrattanamaneewong, MD. are the instructors at Department of Orthopedic Surgery, Faculty of Medicine, Siriraj Hospital, Mahidol University, Bangkok, Thailand.

Received: 22 July 2011 Accepted: 20 July 2012

Published: 20 July 2012

\section{References}

1. Schreiber GB, Busch MP, Kleinman SH, Korelitz JJ: The risk of transfusion transmitted viral infections: the retrovirus epidemiology donor study. N Engl J Med 1996, 334:1685-1690.

2. Delen T, Brostrom LA, Engtrom KG: Autotransfusion after total knee arthroplasty: effects on blood cells, plasma chemistry, and whole blood rheology. J Arthroplasty 1997, 12:517.

3. Gannon DM, Lombardi AV Jr, Mallory TH, et al: An evaluation of the efficacy of postoperative blood salvage after total joint arthroplasty: a prospective randomized trial. J Arthroplasty 1991, 6:109-114.

4. Clements DH, Sculco TP, Burke SW, Mayer K, Levine DB: Salvage and reinfusion of postoperative sanguineous wound drainage: a preliminary report. J Bone Joint Surg Am 1992, 74-A:646-651.

5. Han CD, Shin DE: Postoperative blood salvage and reinfusion after total joint arthroplasty. J Arthroplasty 1997, 12:511-516.

6. Raut W, Stone MH, Wroblewski BM: Reduction of postoperative blood loss after press-fit condylar knee arthroplasty with use of a femoral intramedullary plug. J Bone Joint Surg Am 1993, 75:1356.

7. Juelsgaard P, Larsen UT, Sorensen JV, Madsen F, Soballe K: Hypotensive epidural anesthesia in total knee replacement without tourniquet: reduced blood loss and transfusion. Reg Anesth Pain Med 2001, 26:105-110.

8. Gibbons CE, Solan MC, Ricketts DM, Patterson M: Cryotherapy compared with Robert Jones bandage after total knee replacement: a prospective randomized trial. Int Orthop 2001, 25:250-252.

9. Wang GJ, Hungerford GS, Savory CG, et al: Use of fibrin sealant to reduce bloody drainage and hemoglobin loss after total knee arthroplasty: a brief note on a randomized prospective trial. J Bone Joint Surg Am 2001, 83-A:1503-1505.

10. Levy O, Martinowitz U, Oran A, Tauber C, Horoszwoski H: The use of fibrin tissue adhesive to reduce blood loss and the need for blood transfusion 
after total knee arthroplasty: a prospective, randomized, multicenter study. J Bone Joint Surg Am 1999, 81-A:1580-1588.

11. Sakihara $\mathrm{H}$, et al: A method to control postoperative bleeding after total knee replacement. Seikei-saigaigeka 1988, 31:543-545. in Japanese.

12. Ryu J, Sakamoto A, Honda T, Saito S: The postoperative drain-clamping method for hemostasis in total knee arthroplasty: reducing postoperative bleeding in total knee arthroplasty. Bull Hosp Jt Dis 1997, 56:251-254.

13. Yamada K, Imaizumi T, Uemura M, Takada N, Kim Y: Comparison between 1-hour and 24-hour drain clamping using diluted epinephrine solution after total knee arthroplasty. J Arthroplasty 2001, 16:458-462.

14. Raleigh $E$, Hing $C B$, Hanusiewicz AS, et al: Drain clamping in knee arthroplasty, a randomized controlled trial. ANZ J Surg 2007, 77:333-335.

15. Shen PC, Jou IM, Lin YT, et al: Comparison between 4-hour clamping drainage and nonclamping drainage after total knee arthroplasty. J Arthroplasty 2005, 20:909-913.

16. Tsumara N, Yoshiya S, Chin T, et al: A prospective comparison of clamping the drain or post-operative salvage of blood in reducing blood loss after total knee arthroplasty. J Bone Joint Surg Br 2006, 88-B:49-53.

17. Prasad N, Padmanabhan V, Mullaji A: Comparison between two methods of drain clamping after total knee arthroplasty. Arch Orthop Trauma Surg 2005, 125:381-384

18. Roy N, Smith M, Anwar M, et al: Delayed release of drain in total knee replacement reduces blood loss. A prospective randomized study. Acta Orthop Belg 2006, 72:34-38.

19. Benoni G, Fredin H: Fibrinolotic inhibition with tranexamic acid reduces blood loss and blood transfusion after knee arthroplasty: a prospective, randomized, double blind study of 86 patients. J Bone Joint Surg Br 1996, 78:434-440.

20. Hiippala ST, Strid LJ, Wennerstrand Ml, et al: Tranexamic acid radically decreases blood loss and transfusions associated with total knee arthroplasty. Anesth Analg 1997, 84:839-844.

21. Jansen AJ, Andreica S, Claeys M, et al: Use of tranexamic acid for an effective blood conservation strategy after total knee arthroplasty. $\mathrm{Br} J$ Anaesth 1999, 83:596-601.

22. Good L, Peterson E, Lisander B: Tranexamic acid decreases external blood loss but not hidden blood loss in total knee replacement. $\mathrm{Br} J$ Anaesth 2003, 90:596-5.

23. Camarasa MA, Olle G, Serra-Prat M, et al: Efficacy of aminocaproic, tranexamic acids in the control of bleeding during total knee replacement: a randomized clinical trial. Br J Anaesth 2006, 96:576-582.

24. Veien M, Sorensen JV, Madsen F, et al: Tranexamic acid given intraoperatively reduces blood loss after total knee replacement: a randomized, controlled study. Acta Anaesthesiol Scand 2002, 46:1206-1211

25. Orpen NM, Little C, Walker G, et al: Tranexamic acid reduces early post-operative blood loss after total knee arthroplasty: a prospective randomized controlled trial of 29 patients. Knee 2006, 13:106-110.

26. Hynes M, Calder P, Scott G: The use of tranexamic acid to reduce blood loss during total knee arthroplasty. Knee 2003, 10:375-377.

27. Tanaka N, Sakahashi H, Sato E, et al: Timing of the administration of tranexamic acid for maximum reduction in blood loss in arthroplasty of the knee. J Bone Joint Surg Br 2001, 83:702-705.

28. Ho KM, Ismail H: Use of intravenous tranexamic acid to reduce allogeneic blood transfusion in total hip and knee arthroplasty: a meta-analysis. Anaesth Intensive Care 2003, 31:529-537.

29. Cid J, Lozano M: Tranexamic acid reduces allogeneic red cell transfusions in patients undergoing total knee arthroplasty: results of a meta-analysis of randomized controlled trials. Transfusion 2005, 45:1302-1307.

30. Jou IM, Lai KA, Yang CY, et al: Blood loss associated with total knee arthroplasty. J Orthop Surg (ROC) 1993, 10:213.

31. Kiely N, Hockings M, Gambhir A: Does temporary clamping of drains following knee arthroplasty reduce blood loss?: a randomized controlled trial. Knee 2001, 8:325-327.

32. Risberg B: The response of the fibrinolytic system in trauma. Acta Chir Scand Suppl 1985, 522:245-271

33. Janssens M, Joris J, David JL, Lemaire R, Lamy M: High-does aprotinin reduces blood loss in patients undergoing total hip replacement surgery. Anesthesiology 1994, 80:23-29.

34. Agletti P, Baldini A, Vena LM, et al: Effect of tourniquet use on activation of coagulation in total knee arthroplasty. Clin Orthop 2000, 371:169-177.
35. Klenerman L, Chakrabarti R, Mackie I, Brozovic M, Stirling Y: Changes in haemostatic system after application of a tourniquet. Lancet 1977, 1:970-972.

36. Andersson L, Nilsson IM, Nilehn JE: Experimental and clinical studies on AMCA, the antifibrinolytic active isomer of $p$-aminomethyl cyclohexane carboxylic acid. Scand J Haematol 1965, 2:230

37. Dubber AH, McNicol GP, Douglas AS: Amino methyl cyclohexane carboxylic acid (AMCHA): a new synthetic fibrinolytic inhibitor. $\mathrm{Br} J$ Haematol 1965, 11:237.

38. Nilsson IM: Clinical pharmacology of aminocaproic and tranexamic acids. J Clin Pathol Suppl (R Coll Pathol) 1980, 14:41-47.

39. Hoylaerts M, Lijnen HR, Collen D: Studies on the mechanism of antifibrinolytic action of tranexamic acid. Biochim Biophys Acta 1981, 673:75.

40. Engel JM, Hohaus $T$, Ruwoldt R, et al: Regional hemostatic status and blood requirements after total knee arthroplasty with and without tranexamic acid or aprotinin. Anesth Analg 2001, 92:775-780.

41. Fan $\mathrm{CH}$, Hung $\mathrm{HH}$, Chiu KH: Rate of drainage following total knee replacement. J Orthop Surg (Hong Kong) 2006, 10(1):10-13.

42. Senthil KG, Von Arx OA, Pozo JL: Rate of blood loss over 48 hours following total knee replacement. Knee 2005, 12:307.

43. Benoni G, Bjorkman S, Fredin H: Application of pharmacokinetics data from healthy volunteers for the prediction of plasma concentrations of tranexamic acid in surgical patients. Clin Drug Invest 1995, 10:280-287.

44. Sano M, Hakusui H, Kojima C, Akimoto T: Absorption and excretion of tranexamic acid following intravenous, intramuscular and oral administrations in healthy volunteers. Jpn J Clin Pharmacol Therapeutics 1976, 7:375-382

45. Alvarez JC, Santiveri FX, Romos I, et al: Tranexamic acid reduces blood transfusion in total knee arthroplasty even when a blood conservation program is applied. Transfusion 2008, 48:519-525.

46. Eriksson O, Kjillman H, Pillbrant A, Schannong M: Pharamacokinetics of tranexamic acid after intravenous administration to normal volunteers. Eur I clin Phamacol 1974, 7:375-380.

47. Walzman M, Bonnar J: Effects of tranexamic acid on the coagulation and fibrinolytic systems in pregnancy complicated by placental bleeding. Arch Toxicol Suppl 1982, 5:214-220.

48. Sehat KR, Evans R, Newman JH: How much blood loss is really lost in total knee arthroplasty? Correct blood loss management should take hidden blood loss into account. Knee 2000, 7:151.

doi:10.1186/1471-2474-13-124

Cite this article as: Chareancholvanich et al:: Temporary clamping of drain combined with tranexamic acid reduce blood loss after total knee arthroplasty: a prospective randomized controlled trial. BMC Musculoskeletal Disorders 2012 13:124.

\section{Submit your next manuscript to BioMed Central and take full advantage of:}

- Convenient online submission

- Thorough peer review

- No space constraints or color figure charges

- Immediate publication on acceptance

- Inclusion in PubMed, CAS, Scopus and Google Scholar

- Research which is freely available for redistribution 